\title{
Index to genera and species, Volume 9 (1998)
}

\section{Coleoptera}

Aphodius 79, 80

- contaminatus (Herbst) 79-83

- granarius (L.) 2

- sphacelatus (Panzer) 2

Attagenus smirnovi Zhant. 2

Bolitophagus reticulatus (L.) 66

Carabus 223, 219

- auratus L. 222

- cancellatus I11. 222

- glabratus (Paykull) 217-223

- granulatus L. 222

- nemoralis Müller 219

- problematicus Hbst. 222

- violaceus L. 218, 219, 223

Cis bidentatus (Olivier) 65, 70-77

- boleti (Scopoli) 65, 70-77

- comptus Gyll. 65, 70-74, 76, 77

— hispidus Paykull 65, 70-77

- jacquemarti Mell. 65, 70-77

- lineatocribratus Mell. 65, 70-77

— punctulatus Gyll. 65, 70-72, 76, 77

Cryptophagus corticinus 48

Deleaster dichrous 48

Ennearthron cornutum (Gyll.) 65, 70, 72, 74, 76, 77

Leiodes fracta 48

- lucens 48

Macroplea pubipennis Reuter 168

Magdalis barbicornis (Latr.) 1

Octotemnus glabriculus (Gyll.) 65, 70-77

Orthocis 74

- alni (Gyll.) 74

- festivus (Panzer) 65, 71, 72, 74-77

— linearis (Sahlb.) 74

Pterostichus cupreus L. 222, 223

— melanarius Ill. 223

Pullus mundus 89

Rhizophagus ferrugineus 48

Ropalodontus strandi Lohse 65, 70, 72-77

Scymnus agnavus Mader 87

— auritus Thunberg 85,87

- centrorubicus Mader 86, 87

- couturieri Chazeau 85, 94

- flavipubens Mader 94

- frontalis 85

- fuscus Fürsch 94

- gnavus Weise 87

— inaequalis Mader 90, 94
- majeri Fürsch 85, 94

- majusculus Mader 90, 92, 94

- mundus Mader 85, 87, 90

- nasti Fürsch 94

- nigrosellatus Mader 85, 87, 89, 90

- plutonus Mulsant 87

- propiptoides Sicard 87

Scymnus (Pullus) 85

- $(P$.$) auritoides Fürsch sp. n. 85-87$

- (P.) fomosipennis Fürsch sp. n. 85, 87, 88

- $(P$.) nigropectus Mader 85, 87, 90, 91, 94

-(P.) siphonofissus Fürsch sp. n. 85, 93, 94

Stagetus borealis 48

Stenotrachelus aeneus (Paykull) 4

Stereum 74

Sulcacis affinis (Gyll.) 65, 70-74, 76, 77

- fronticornis (Panzer) 65, 70, 72, 76, 77

Tenebrio molitor Linnaeus 219

Thalycra fervida 48

Trypodendron lineatum (Olivier) 77

\section{Diptera}

Aenigmatias lubbocki (Verrall) 1

Asianochlorops Kanmiya 159, 168

Assuania Becker 168

— distinguenda Frey 154

Bryomyia Kieffer 147, 148, 150, 151

— amurensis Mamaev \& Økland sp. n. 147-149, 151

— apsectra Edwards 147, 148, 150, 151

— bergrothi Kieffer 147-149, 151

- cambrica Edwards 147

- gibbosa (Felt) 147, 149, 151

— incisa Mamaev 147-151

- longipennis Mamaev 147, 148, 150

- multispinata Berest 147,150

- producta Pritchard 147-151

- trifida Edwards 147

Capnoptera Loew 153

Cervuatina Berest 147

Chironomus 225, 231, 232, 234

sp. "2n = 6, Lokka" (Michailova) 225, 226, 230, 232, 233

— anthracinus Zetterstedt 232

- brevidentatus Hirvenoja \& Michailova sp. n. 225-234

- cingulatus Meigen 226

- cucini Webb 231

- entis Shobanov 234

- fraternus Wülker 231 
- islandicus Kieffer 230, 232, 233

- jonmartini Lindeberg 231

- longistylus Goetghebuer 231, 232

— major Wuilker \& Butler 225, 226, 231

- neocorax Wülker \& Butler 225-233

- plumosus Linnaeus 234

- prior Butler 231

- tardus Butler 225, 226, 231

- venustus Staeger 231, 232

Chlorops Meigen 153-155, 158-160, 168

—anthracophagoideus Strobl 154, 157, 160, 162, 166, 179

- bipunctus Duda 167

- brevimanus Loew 156, 157, 159, 162, 167, 180

_ calceatus Meigen 155, 157, 166, 167 169, 172, 173, 180

— centromaculatus (Duda) 154, 155, 157, 160, 167, 183

- certimus Adams 169

— citrinellus Zetterstedt 155, 157, 162, 167, 174, 183

- crassipalpis Smimov 154, 155, 157, 161, 167, 169, 183

- distinguendus Frey 155, 157, 159, 160, 168, 183

- fasciatus Becker 156, 157, 163

- figuratus Zetterstedt 154, 156, 157, 163, 164, 173, 174

- finitimus Becker 156, 157, 161, 163

- freyi Duda 153, 171

-frontosus Meigen 156, 157, 163, 174

- geminatus Meigen 154, 157, 164, 165, 168, 173, 179

- gigantea 172

- gracilis Meigen 155, 157, 168, 177

- hypostigma Meigen 155, 157, 162, 168, 180

— interruptus Becker 156, 157, 164

— kirigaminensis Kanmiya 168

- laetus Meigen 154, 157, 163, 169, 179

- laevicollis Becker 153, 154, 171

- lateralis Haliday 159

- lenis Becker 168

- leymi Nartshuk 168

- major Frey 172

- meigenii Loew 170

— nigripalpis (Duda) 154, 157, 163, 169

— novakii Strobl 156, 157, 163, 165, 174

—obscurellus (Zetterstedt) 155-157, 164, 169, 177

— pallifrons Strobl 172

- palpatus Smirnov 157, 160

— pannonicus Strobl 156, 157, 161, 163, 174

_ planifrons Loew 154, 156, 157, 164, 169, 173, 181

- pumilionis (Bjerkander) 155, 157, 162, 163, 170, 180

- ringens Loew 155, 157, 161, 170, 177

- riparius Smimov 156, 157, 164

- rossicus Smirnov 154, 156, 157, 161, 165, 17 0, 178

- rufescens Oldenberg 154, 156, 157, 159, 161, 165, 166,

$170-172,181$

- rufinus Zetterstedt 156, 157, 161, 171, 174, 182

— scalaris Meigen 156, 157, 161, 171, 182

— scutellaris Zetterstedt 156-159, 162, 171, 172, 175, 178

— serenus Loew 154, 155, 157, 160, 164, 172, 177

- speciosa var. freyi 171

- speciosus Meigen 156, 157, 159, 165, 166, 170-172, 178

— strigulus (Fabricius) 156, 157, 163, 174
- triangularis Becker 154, 169

- troglodytes (Zetterstedt) 156, 157, 166, 167, 172, 182

- varsoviensis Becker 156, 157, 159, 162, 173, 181

—zernyi (Duda) 154, 157, 163, 173, 179

—zonulatus Wahlgren 154, 156, 157, 164, 165, 168, 173175,182

Clinorrhytis Kieffer 212

Corynoneura 235

Cryptoxylomyia Mamaev gen. n. 211-213

- excavata (Mamaev) 213

Cynomya mortuorum (Linnaeus) 2

Dasineura marginemtorquens (Larsson \& Strong) 96, 99, 100

- tetensi 95-101

Dicrotendipes nervosus (Staeger) 234

Discachaeta pumila (Meigen) 2

Ephydra macellaria alandica Frey 168

Epichlorops Becker 153, 154, 159

— puncticollis (Zetterstedt) 156, 157, 159, 173, 178

Eurosta solidaginis 96

Heterogenella Mamaev 147

Kronomyia Felt 212

Mayetiola destructor (Morril) 96, 99, 100

Melanum Becker 153, 154, 159

- laterale (Haliday) 156, 157, 159, 160, 173, 181

Melinda viridicyanea (Robineau-Desvoidy) 2

Meromyza Meigen 154, 155

Musca pumilionis Bjerkander 158

Opomyza nigriventris Loew 156

Oscinis bipunctifrons var. fennica 167

- bipunctus Duda 154

- freyi Duda 154, 171

- geminata 167

- longicornis Fallén 159

- puncticollis Zetterstedt 159

- scutellaris Zetterstedt 153

Parectecephala Becker 153, 158, 159

Parectecephala longicornis 159

Parwinnertzia Felt 212

Platycheirus jaerensis Nielsen 1

- occultus Goeldlin, Maibach \& Speight 2

Pollenia hungarica Rognes 2

Potthastia longimanus Kieffer 234

Rhipidoxylomyia Mamaev 211, 212

- excavata Mamaev 213

Sclerophallus Beschovski 159

Skuhraviana Mamaev 147

Sylvenomyia Mamaev \& Zaitzev gen, n. 211, 212

- sueciae Mamaev \& Zaitzev sp. n. 211, 212

Tanytarsus 235

Winnertzia Rondani 212

\section{Heteroptera}

Agraphopus suturalis Reuter 240

Amnestus Dall 5, 7 
- pusillus Uhler 5, 7

- pusio (St'1) 7

- raunoi Lis sp. n. 5-7

Amphiareus obscuriceps (Poppius) 240

Anthocoris minki pistaciae Wagner 239

— pilosus (Jakovlev) 239

Aradus 3

- betulae (Linnaeus) 4

- laeviusculus Reuter 3, 4

- lugubris Fallén 4

Brachycarenus languidus (Horwáth) 240

- tigrinus (Schilling) 240

Centrocoris inflaticeps Kiritshenko 240

- volxemi (Puton) 240

Chartoscirta elegantula longicornis (Jakovlev) 238

Chilocoris Mayr 7

— repetitus (Uhler) 7

Chorosoma gracile Josifov 241

- schillingi (Schummel) 240

Coreus marginatus (Linnaeus) 240

Coriomeris validicornis Jakovlev 240

— vitticollis Reuter 240

Corixa affinis Leach 238

Corizus hyoscyami (Linnaeus) 240

Cymatia rogenhoferi (Fieber) 238

Euglenes pygmaeus (Degeer) 4

Gerris (Gerris) costae (Herrich-Schäeffer) 238

- (G.) thoracicus Schummel 238

Gonocerus acuteangulus (Goeze) 240

Haploprocta pustulifera (Stål) 240

Hebrus pilipes Kanyukova 238

Hesperocorixa occulta (Lundblad) 238

Himacerus (Aptus) maracandicus (Reuter) 239

Hydrometra stagnorum (Linnaeus) 238

Liorhyssus hyalinus (Fabricius) 240

Lygocoris contaminatus 48

— viridis 48

Lygus rhamnicola 48

Maccevethus caucasicus (Kolenati) 240

- corsicus Signoret 240

Microporus Uhler 7

— obliquus Uhler 7

Nabis (Aspilaspis) indicus (Stål) 239

- (A.) viridulus Spinola 239

- (Halonabis) sareptanus Dohrn 239

- (Nabis) palifer Seidenstïcker 239

- (N.) pseudofredus transcaspicus Remane 239

- (N.) punctatus A.Costa 239

- (Tropiconabis) capsiformis Germar 239

Notonecta maculata Fabricius 238

- viridis Delcourt 238

Orius (Dimorphella) albidipennis (Reuter) 239

- (Heterorius) horvathi (Reuter) 238

- (H.) laticollis discolor (Reuter) 239

- (H.) vicinus (Ribaut) 239

- (Orius) laevigatus Fieber 239

- (O.) niger (Wolff) 239

Pediacus fuscus Erichson 4
Phyllomorpha lacerata Herrich-Schäffer 240

Phytocoris longipennis 48

Plagiognathus albipennis 48

Pteropyrum aucheri 240

Pyrrhocoris apterus (Linnaeus) 241

Rhopalus (Rhopalus) curtulus (Kiritshenko) 240

- (R.) lepidus Fieber 240

- (R.) parumpunctatus Schilling 240

- (R.) subrufus (Gmelin) 240

Saldula pallipes (Fabricius) 239

- setulosa (Puton) 239

Sehirus Amyot \& Serville 7

- cinctus (Palisot de Beauvois) 7

Sigara (Pseudovermicorixa) nigrolineata (Fieber) 238

- (Sigara) seistanensis (Distant) 238

- (Tropocorixa) hoggarica (Poisson) 238

- (Vermicorixa) lateralis (Leach) 238

Sphaeriestes stockmanni (Biström) 4

Stictopleurus pictus (Fieber) 240

- subtomentosus (Rey) 240

Syromastes rhombeus (Linnaeus) 240

Temnustethus (Ectemnus) reduvinus parilis (Horváth) 239

Velia affinis affinis Kolenati 238

\section{Homoptera}

Alnetoidia alneti 48

Cixidia confinis Zetterstedt 168

Cosmotettix evanescens Ossiannilsson 168

Empoasca kontkaneni 48

- smaragdula 48

- vitis 48

Linnavuoriana decempunctata 48

- sexmaculata 48

Macustus grisescens 48

Populicerus laminatus 48

Stenocranus major (Kirschbaum) 1

\section{Hymenoptera}

Bodedia 104

Cremastus Gravenhost 215

Formica fusca Linnaeus 1

- transkaucasica Nasonov 1

Ichneumon domator Poda 8

- gladiator Scopoli 8

- moderator 215

Leptocampoplex 215

_ cremastoides (Holmgren) 215, 216

Lissaspis Townes 103, 104, 113, 114

— areolata Jussila sp. n. 103, 105-107

- argentina Jussila sp. n. 103, 105-108

— brasiliensis Jussila sp. n. 103, 105-108

—exigua Jussila sp. n. 103, 106, 109

- flagellata Jussila sp. n. 103, 105, 106, 109, 110 
— inca Jussila sp. n. 103, 105, 106, 110

- laevigata Townes 103, 104, 106, 109, 110, 112

- longistriata Jussila sp. n. 103, 105, 106, 110-112

- obscura Jussila sp. n. 103, 106, 112

Macrus Gravenhorst 215

Mesostenus gladiator (Scopoli) 8

Metopius harpyiae Clément 52

Nemeritis cremastoides Holmgren 215

Paraphylax 104

- hiatus Townes 104

Phaedroctonus 215

- cremastoides 215

- moderator (Linnaeus) 215, 216

Porizon Fallén 215

- moderator 215

- sensu Townes 215

Singalissaspis gen. n. 103, 104, 112, 113

- tricolor Jussila sp. n. 103, 112, 113

Stenarella Szépligeti 8

- domator (Poda) 8

- d. cruentator (Klug) 8

-d. domator (Poda) 8

Tylopius Townes 52

\section{Lepidoptera}

Acleris emargana (Fabricius) 204

- hastiana 208

- idonea Razowski 197, 204, 208

Acornutia nana 199

Adoxophyes orana (Fischer v. Rösslerstamm) 204

Aethes alatavica (Danilevsky) 201, 208

- decens Razowski 201, 208

- deutschiana (Zetterstedt) 201, 208

- margarotana (Duponchel) 201

- moribundana (Staudinger) 201, 208

- williana (Brahm) 201

Aglais urticae (L.) 13, 14, 16, 17

Agrochola 26

- litura (Linnaeus) 22, 26, 33

- lychnidis (Denis \& Schiffermüller) 19, 20, 22, 23, 26,

29, 33-35

- macilenta (Hübner) 20

- meridionalis (Staudinger) 22, 26, 33

- orientalis Fibiger 19-22, 26, 28, 33-35

Agrodiaetus amandus (Schn.) 14, 16

Agrotis ipsilon (Hufnagel) 20

- segetum (Denis \& Schiffermüller) 20

- trux (Hübner) 20

Allophyes 26

- alfaroi Agenjo 25

- asiatica (Staudinger) 19, 20, 31, 34, 36

- a. cypriaca Boursin 24, 25, 33

- oxyacanthae (Linnaeus) 25, 33

Ammoconia aholai Fibiger 19, 20, 23, 24, 26, 30, 33-36

- caecimacula (Denis \& Schiffermüller) 24, 33
- senex (Geyer) 23, 24, 33

Amphicoecia adamana (Kennel) 200, 203

Ancylis badiana (Denis \& Schiffermüller) 205

- comptana (Frölich) 205

- geminana (Donovan) 205

- kenneli Kuznetsov 205

- myrtillana (Treitschke) 205

- tineana (Hübner) 205

- unguicella (Linnaeus) 205

Aneuxanthis disjuncta 203

Aphantopus hyperantus (L.) 13, 14, 16

Aphelia aglossana Kennel 200, 203, 208

— disjuncta (Filipjev) 203, 208

- viburnana (Denis \& Schiffermüller) 203

Aporia crataegi (L.) 14

Aporophyla australis (Boisduval) 20

- canescens (Duponchel) 20

- nigra (Haworth) 20

Apotomis capreana (Hübner) 204

- semifasciana (Haworth) 204

- sororculana (Zettertstedt) 204

Archips betulanus (Hübner) 204

- decretana Treitschke 204

Argynnis paphia 16

Argyroploce aquilonana Karvonen 138

- bowmanana McDunnough 141

- dalecarliana 139

- heinrichana McDunnough 138

- hyperboreana Karvonen 138

- minaki McDunnough 141

- palustrana 140

- vulgana McDunnough 137, 138

Arenosana 199

Aricia artaxerxes (Fabr.) 14

Asketria kenteana (Staudinger) 202, 207

Aterpia sieversiana (Nolcken) ssp. quadrimaculana (Snel-

len) 204

Autographa gamma (Linnaeus) 20

Bactra furfurana (Haworth) 204

- lacteana (Caradja) 204

- robustana (Christoph) 204

Ballota sp. 198

Brenthis ino (Rott.) 14, 16, 17

Cadra Walker 143

Callophrys rubi (L.) 14, 16, 17

Capricornia boisduvaliana (Duponchel) 205

Capua vulgana (Frölich) 204

Carterocephalus silvicola (Meig.) 14

Celastrina argiolus (L.) 14

Celypha cespitana (Hübner) 204

- flavipalpana (Herrich-Schäffer) 204

- rufana (Scopoli) 204

Chionodes fumatella 43

Chloroclysta citrata 43

Choristoneura albaniana (Walker) 204

- (lapponana Tngstr.) 204

Chrysodeixis chalcites (Esper) 20

Cidaria Treitschke 186, 191 
- bicolorata ab. guttata Huene 187

- b. ab. maritima Strand 187

- b. dahurica Staudinger 187

- fulvata (Forster) 188, 190

- (Plemyria) rubiginata Prout 187

Clepsis aerosana (Lederer) 200, 203

- crispinana (Kennel) 200, 203, 208

- danilevskyi Kostjuk 200, 203, 208

- neglectana (Herrich-Schäffer) 203

- pallidana (Fabricius) 203

- praeclarana (Kennel) 200, 203, 208

- rogana (Guenée) 200, 203, 208

- rurinana (Linnaeus) 203

- semialbana Guenée 203

- senecionana (Hübner) 203

- tannuolana Kostjuk 200, 203, 208

Clossiana euphrosyne (L.) 14, 16

- selene (D. \& S.) 14, 16

Cnephasia novickii Razowski 200, 203

Cochylidia moguntiana (Rössler) 199

Cochylimorpha 199

— arenosana Kuznetsov, Jalava \& Kullberg sp. n. 197, 199

201,203

— asiana (Herrich-Schäffer) 200, 201, 208

- cultana (Lederer) 201

- discolorana (Kennel) 201, 208

- emiliana (Kennel) 200, 203, 208

- fucatana (Snellen) 200, 201

- hedemanniana (Snellen) 199

- nodulana (Möschler) 201, 208

—obliquana (Eversmann) 200, 201, 208

- perturbatana (Kennel) 201, 208

- pyramidana (Staudinger) 203, 208

- richteriana (Fischer v. Röslerstamm) 201

- woliniana (Schleich) 201

-w. ssp. luteola 209

Cochylis nana (Haworth) 199

Coenonympha glycerion (Brkh.) 14, 16, 17

- pamphilus (L.) 14

Colias palaeno (L.) 14

Condica viscosa (Freyer) 20

Conistra rubricans Fibiger 20, 26

- vaccinii (Linnaeus) 23, 33

Cosmiotes Clemens 53, 55, 56

Croesia bergmanniana (Linnaeus) 204

Cucullia Schrank 25

- calendulae Treitschke 20, 26

Cyaniris semiargus (Rott.) 14, 16, 17

Cydia medicaginis (Kuznetsov) 207

— nigricana (Fabricius) ssp. asiatica (Kuznetsov) 207

- oxytropidis (Martini) 207

- populana (Busck) ssp. seductana (Kuznetsov) 207

- strobilella (L.) 215

Dichrorampha alaicana (Rebel) 202, 207

- cinerascens (Danilevsky) 202, 207

- gueneeana Obraztsov 207

- incognitana (Kremky \& Maslowski) 202, 207

- petiverella (Linnaeus) 207
- plumbana (Scopoli) 207

Dicranoctetes Braun 55, 57, 59

Djakonovia accuratana (Kennel) 203

- praeclarana ssp. fucosana Kennel 204

Dryobotodes carbonis (F.Wagner) 20

Dysstroma Hübner 185, 186

- truncatum (Hufnagel) 190

Eana argentana (Clerck) 203

- osseana (Scopoli) 203, 208

Elachista Treitschke 53, 55-57, 62

— abiskoella Bengtsson 63

- albrechti Kaila sp. n. 53, 59-61

— bifasciella 55, 58

- freyerella 53, 58

- heteroplaca Meyrick 53-55, 57-59, 61

- leucosyrma (Meyrick) 61, 62

- lorigera (Meyrick) 54, 56, 58, 59

_ planicara Kaila sp. n. 53, 54, 57-59

- tetragonella 53, 61, 62

Endothenia gentianaeana (Hübner) 205

- nigricostana (Haworth) 205

- quadrimaculana (Haworth) 205

Ephestia Guenée 143, 144, 146

- animella Nupponen \& Junnilainen sp. n. 143-146

- columbiella 143

- disparella Ragonot 144, 146

- elutella (Hübner) 143

— inquietella Zerny 144, 146

- kuehniella (Zeller) 143

- parasitella Staudinger 144, 146

- p. unicolorella Staudinger 144

- personata Gerasimov 143, 144

Epiblema arctica Miller 137

- foenellum (Linnaeus) 206

- graphanum (Treitschke) 206

- simplonianum (Duponchel) 137

Epinotia bilunana (Haworth) 206

- caprana (Fabricius) 206

- contrariana (Christoph) 206

- demarniana (Fischer v. Röslerstamm) 206

- nisella (Clerck) 206

- solandriana (Linnaeus) 206

Erebia ligea (L.) 14, 16

Eriopsela mongunana Kotsjuk 207, 208

- quadrana (Hübner) 207, 208

Eublemma ostrina (Hübner) 20

Eucosma abacana (Erschoff) 202, 206

- aemulana (Schläger) 206

- apocrypha Falkovich 202, 206

- argentifera Razowski 197, 202, 206, 208

- aspidiscana (Hübner) 206

- aurantiradix Kuznetsov 206

- brigittana (Kennel) 206

- decolorana (Freyer) 206

- lacteana (Treitschke) 206

- lignana (Snellen) 202, 206

- lugubrana (Treitschke) 202, 206

- metana (Kennel) 206 
- muguraxana Kostjuk 202, 206, 208

- obacana (Erschoff) 206

- oculatana (Kennel) 206

- paetulana (Kennel) 202

- p. (Kennel) ssp. mirana (Caradja) 206

- pergratana (Rebel) 206, 208

- tetraplana (Möschler) 202, 206

- tundrana (Kennel) 20

Eudonia 144

Eulithis populata 43

Eumedonia eumedon (Esper) 14, 16, 17

Eupithecia pusillata 43

Eupneusta bradley 55

Eurodryas aurinia 16

Fabrigiana adippe (D. \& S.) 14, 16

— niobe (L.) 14

Falseuncaria ciliella (Hübner) 199

- lechriotoma Razowski 197, 199, 208

- ruficiliana (Haworth) 199

Fixsenia pruni (L.) 14

Furcula furcula 52

Geometra rubiginata Denis \& Schiffermüller 185-187

Gibberifera simplana (Fischer v. Röslerstamm) 205

Glaucopsyche alexis (Poda) 14, 17

Gonepteryx rhamni (L.) 13, 14, 16

Grapholita caecana (Schläger) 207

- jungiella (Clerck) 207

- orobana (Treitschke) 207

- pallifrontana (Zeller) 207

Gypsonoma minutana (Hübner) 205

- nitidulana (Zeller) 205

- sociana (Haworth) 205

Harpyia furcula 52

Hedya atropunctana 139

- ochroleucana (Frölich) 204

— salicella (Linnaeus) 204

- separatana 139

Heliothis nubigera Herrich-Schäffer 20

- peltigera (Dennis \& Schiffermüller) 20

- viriplaca (Hufn.) 2

Heodes virgaureae (L.) 14, 16

Homoeosoma 144

Hypena lividalis (Hübner) 20

Hypodryas maturna (L.) 14, 16, 17

Hydriomena 185, 186

Inachis io (L.) 14, 16

Ipimorpha subtusa 43

Issoria lathonia 16

Larentia bicolorata Hufn. 191

— b. ab. completa Rebel 187

— b. ab. diadelphata Stauder 187

— b. ab. peralbata Stauder 187

- b. ab. rosarium Stauder 187

Lasiommata maera (L.) 14, 16

- petropolitana 16

Laspeyresia strobilella (L.) 215

Leptidea sinapis (L.) 14, 16, 17

Limenitis populi (L.) 14,17
Lithophane lapidea (Hübner) 20

Lobesia subherculeana Filipjev 202, 205

Lycaeides idas (L.) 14, 16, 17

Lycaena phlaeas (L.) 14, 16

Melanthia bicolorata ab. fumosa Prout 187

Mellicta athalia (Rott.) 14, 16

Metendothenia atropunctana (Zetterstedt) 139

- separatana (Kearfott) 139

Mirana 206

Mixodia intermistana Clemens 138

Mythimna l-album (Linnaeus) 20

- languida (Walker) 20

- loreyi (Duponchel) 20

- unipuncta (Haworth) 20

Noctua pronuba Linnaeus 20

Nola aegyptiaca Snellen 20, 26

Notocelia cynosbatella (Linnaeus) 206

- incarnatana (Hübner) 206

Nycteola columbana Turner 20

Nymphalis antiopa (L.) 14, 16, 17

Ochlodes venatus (Br. \& Gr.) 13, 14, 16

Ocnogyna loewii (Zeller) 20

Olethreutes 137

- aquilonanus (Karvonen) 137, 138

- a. helvomaculanus Kostjuk 138

- bowmananus (McDunnough) 141

- concretanus (Wocke) 140

- costimaculanus (Fernald) 139-141

- dalecarlianus (Guenée) 139, 141

- dissolutanus (Stange) 141

- exaridanus Kuznetsov 140, 141

- heinrichana McDunnough 138

- heinrichanus (McDunnough) 137, 138

- hyperboreanus (Karvonen) 137, 138

— intermistanus (Clemens) 137, 139

- kennethana McDunnough 140

- kennethanus McDunnough 137, 140

- kononenkoi Kuznetsov 137, 138

- ledianus (L.) 141

- mengelanus (Fernald) 141

- minaki McDunnough 141

- obsoletana 140

- obsoletanus (Zetterstedt) 137, 140

- palustranus (Lienig \& Zeller) 140

- schaefferanus (Herrich-Schäffer) 137

- septentrionanus (Curtis) 137, 139

- turfosanus (Herrich-Schäffer) 137, 138, 139

Orgyia dubia ('l'auscher) 20

Orthotaenia septentrionana Curtis 139

- undulana (Denis \& Schiffermüller) 204

Palaeochrysophanus hippothoe (L.) 14, 16

Pandemis cerasana (Hübner) 203

- ribeana Hübner 203

Papilio machaon (L.) 14

Paradrina flavirena (Guenée) 20

Pelochrista arabescana (Eversmann) 202, 208

- a. (Eversmann) ssp. ornamentana (Rebel) 207

- chanana (Staudinger) 202, 206, 208 
- danilevskyi Kotsjuk 202, 207, 208

- discquei (Kennel) 207, 208

- jodocana (Kennel) 207, 208

- tholera Falkovich 207, 208

- umbraculana (Eversmann) 202, 207

Penthina atro-punctana Zetterstedt 139

- concretana Wocke 140

- concretanus (Wocke) 140

- costimaculana Fernald 141

- enervana (Erschoff) 138

Peridroma saucia (Hübner) 20

Periploca Braun 54

Phalaena abbreviata Haworth 187

Phalaena bicolorata Hufnagel 187

Phalonidia minimana (Caradja) 201

- walsinghamana Pierce 201

Phiaris 140

- bipunctana (Fabricius) 205

- inquietana (Walker) 202, 208

- inquietana (Walker) ssp. hepialana (Kennel) 205

- metallicana (Hübner) 205

- obsoletana (Zetterstedt) 205

- obsoletanus 140

- palustrana 140

- schaefferana 139

- stibiana (Guenée) 205

- tiedemanniana (Zeller) 205

- turfosana 139

Philedone gerningana (Denis \& Schiffermüller) 204

Phtheochroa 199

- inopiana (Haworth) 203

- pistrinana (Erschotf) 200, 203

- vulneratana (Zetterstedt) 203, 208

Pieris brassicae (L.) 14, 16, 17

- napi (L.) 13, 14, 16, 17

- rapae (L.) 14, 16, 17

Pilosella officinarum 11

Platyphyllis Meyrick 53, 62

- leucosyrma Meyrick 53, 61-63

Plebejus argus (L.) 14

Plemyria Hübner 185-187, 190, 191

- bicolorata Hufnagel 185

— b. ab. bipunctata Hannemann 187

- georgii (Hulst) 185-191, 193

- japonica Inoue 186, 190

— rubiginata (Denis \& Schiffermüller) 185-188, 190-192,

194-196

- r. ab. albovittata Cockayne 187

- r. ab. pallidaria Cockayne 187

- r. ab. semifumosa Cockayne 187

- r. ab. tenebra Inoue 187, 188

- r. dahurica Staudinger 186, 188, 190

- r. japonica Inoue 187, 188, 190

- r. ssp. benesignata Bames \& McDunnough 186, 190
Polygonia c-album (L.) 14, 16

Polymixis aphrodite Fibiger 20, 26

- leuconota (Herrich-Schäffer) 25, 26, 33

- rufocincta (Geyer) 20, 25, 26, 33

- trisignata (Ménétriés) 19, 20, 25, 26, 32-34, 36

Polyommatus icarus (Rott.) 14, 16

Ptilodoxa 53, 57

- lorigera Meyrick 53, 57

Ptycholoma lecheanum (Linnaeus) 204

-l. ssp. circumclusanum (Christoph) 204

Ptycholomoides aeriferanus (Herrich-Schäffer) 204

Pyrgus alveus 16

- malvae (L.) 14

Retinia perangustana (Snellen) 205

Rhopoboata naevana 44

Rudisociaria expeditana (Snellen) 202, 204

Selenodes dalecarliana (Guenée) 139

Sericoris mengelana Fernald 141

- palustrana Lienig \& Zeller 140

- schaefferana Herrich-Schäffer 139

- turfosana Herrich-Schäffer 138

Speyeria aglaja (L.) 14, 16

Spodoptera cilium (Guenée) 20

- exigua (Hübner) 20

- littoralis (Boisduval) 20

Syricoris lacunana (Denis \& Schiffermüller) 205

— rivulana (Scopoli) 205

Thecla betulae (L.) 14, 17

Thera 186

- georgii benesignata Barnes \& McDunnough 188, 189

- variata (Denis \& Schiffermüller) 187, 190

Thymelicus lineola (Och.) 13, 14, 16

Tia enervana (Erschoff) 137, 138, 202, 204

Trichoplusia ni (Hübner) 20

- vittata (Wallengren) 20, 26

Vacciniina optilete (Knoch) 14,16

Vanessa atalanta (L.) 14

- cardui (L.) 14, 16

Xerocnephasia rigana (Sodoffsky) 203

Xylena exsoleta (Linnaeus) 20

Zeiraphera griseana (Hübner) 205

- ratzeburgiana 43

Zerene bicolorata ab. plumbata Curtis 187

\section{Orthoptera}

Chortippus brunneus brevis Klingstedt 168

\section{Trichoptera}

All Finnish species presented 115-135 